\title{
Tendência Temporal da Mortalidade por Doenças Isquêmicas do Coração no Nordeste Brasileiro (1996-2016): Uma Análise Segundo Gênero e Faixa Etária
}

\author{
Temporal Trend of Mortality Due to Ischemic Heart Diseases in Northeastern Brazil (1996-2016): An Analysis \\ According to Gender and Age Group
}

Gibson Barros de Almeida Santana, ${ }^{1}$ Thiago Cavalcanti Leal,, João Paulo Silva de Paiva, ${ }^{1}$ Leonardo Feitosa da Silva, ${ }^{1}$ Lucas Gomes Santos, ${ }^{1}$ Tatiana Farias de Oliveira, ${ }^{1}$ Rodrigo da Rosa Mesquita, ${ }^{1}$ Jéssica Alves Gomes, ${ }^{1}$ Carlos Dornels Freire de Souza, ${ }^{10}$ Amanda Karine Barros Ferreira Rodrigues ${ }^{1 \oplus}$

Universidade Federal de Alagoas, ${ }^{1}$ Maceió, AL - Brasil

\section{Resumo}

Fundamentos: As doenças isquêmicas do coração (DIC) são a primeira causa de mortes dentre as doenças cardiovasculares (DCV).

Objetivo: Descrever o perfil sociodemográfico e analisar tendência da taxa de mortalidade por DIC, segundo sexo e por faixa etária, nos estados da região Nordeste do Brasil, 1996-2016.

Métodos: Estudo ecológico envolvendo a mortalidade por DIC nos estados do Nordeste. Variáveis analisadas: sexo, idade, escolaridade, estado civil, categoria do CID-10 e estado de residências. Foram calculadas taxas brutas e padronizadas. Os dados de óbitos foram coletados do Sistema de Informações sobre Mortalidade; e os dados populacionais, do Instituto Brasileiro de Geografia e Estatística (IBGE). Nas análises temporais, utilizou-se o modelo de regressão por pontos de inflexão, com cálculo do percentual de variação anual (APC, Annual Percent Change) e do percentual de variação médio do período (AAPC, Average Annual Percent Change). Considerou-se intervalo de confiança de 95\% e significância de $5 \%$.

Resultados: Registrou-se 405.916 óbitos por DIC na região Nordeste durante o período estudado. O perfil de óbitos caracteriza-se por homens $(n=229.006 ; 56,42 \%)$, idosos $(n=301.379 ; 74,25 \%)$, raça/cor parda $(n=197.936 ; 48,76 \%)$, fundamental ou $<4$ anos na escola $(n=232.599 ; 57,30 \%)$ e casados $(n=179.599 ; 44,25 \%)$. Houve destaque incomum para o aumento na taxa de incremento anual na faixa etária de adolescentes (AAPC: 5,2\%, $\mathrm{p}<0,01$ ). A taxa de mortalidade regional padronizada cresceu de 30,7/100 mil habitantes, em 1996, para 53,8/100 mil, em 2016 (AAPC:2,8\%; p<0,01). Todos os nove estados apresentaram tendência estatisticamente significante de crescimento, com ênfases para o Maranhão (AAPC:7,6\%; p<0,01) e o Piauí (AAPC:6,0\%; $<<0,01$ ).

Conclusão: O perfil prevalente observado foi de homens, idosos, raça/cor parda, baixa escolaridade e casados. A mortalidade por DIC apresentou tendência de crescimento em todos os estados, ainda que com padrão desigual entre as unidades federadas.

Palavras-chave: Isquemia Miocárdica/mortalidade; Epidemiologia; Fatores Socioeconômicos; Mortes; Análise Estatística; Demografia; Saúde Pública.

\footnotetext{
Abstract

Background: Ischemic heart disease (IHD) is the leading cause of death among cardiovascular diseases (CVD).

Objective: To describe the sociodemographic profile and analyze the trend in the mortality rate due to IHD, according to sex and by age group, in the states of the Northeast region of Brazil, from 1996 to 2016.

Methods: Ecological study involving IHD mortality in the northeastern states. Variables analyzed: sex, age, education, marital status, ICD-10 category and state of residence. Crude and standardized rates were calculated. Death data were collected from the Mortality Information System (SIM) and population data from the Brazilian Institute of Geography and Statistics (IBGE). In temporal analyzes the regression model by inflection points was used, with the calculation of annual percent change (APC) and average annual percent change of the period (AAPC). A 95\% confidence interval and a significance level of $5 \%$ were considered.

Correspondência: Carlos Dornels Freire de Souza •

Universidade Federal de Alagoas - Campus Arapiraca - Medicina - Av. Manoel Severino Barbosa. CEP 57309-005, Arapiraca, AL - Brasil

E-mail: carlos.freire@arapiraca.ufal.br

Artigo recebido em 20/03/2020, revisado em 31/05/2020, aceito em 05/08/2020
}

DOI: https://doi.org/10.36660/abc.20200222 
Results: 405916 deaths due to IHD were registered in the northeast region during the study period. The death profile is characterized by men ( $n=229006 ; 56,42 \%)$, elderly $(n=301379 ; 74,25 \%)$, race/color brown $(n=197936 ; 48,76 \%)$, elementary or $<4$ years at school $(n=232599 ; 57,30 \%)$ and married $(n=179599 ; 44,25 \%)$. There was an unusual highlight to the increase in the annual growth rate in the age group of adolescents (AAPC: 5,2\%, $p$ <0.01). The standardized regional mortality rate grew from 30,7 per 100,000 inhabitants in 1996 to 53.8 per 100,000 in 2016 (AAPC 2.8\%; $p<0.01$ ). All nine states presented a statistically significant growth trend, with emphasis on Maranhão (AAPC 7,6\%; $<<0.01$ ) and Piauí (AAPC 6,0\%; $p<0.01$ ).

Conclusion: The prevalent observed profile was male, elderly, race/color brown, low education level and married. Mortality due to IHD presented an upward trend in all states, although with an uneven pattern among the federated units.

Keywords: Myocardial Ischemia/mortality; Epidemiology; Socioeconomic Factors; Deaths; Statistical Analysis; Demography; Public Health.

Full texts in English - http://www.arquivosonline.com.br

\section{Introdução}

O rápido envelhecimento populacional, o processo de urbanização acelerado e as mudanças socioeconômicas contínuas impactaram no estilo de vida dos indivíduos nas últimas décadas, modificando o perfil epidemiológico..$^{1-3}$ Nesse cenário, as doenças crônicas não transmissíveis (DCNT) doenças cardiovasculares (DCV), cânceres, doenças respiratórias crônicas e diabetes melito (DM) - ganharam espaço no contexto epidemiológico e social, constituindo um problema global de saúde responsável por 40 milhões de mortes anuais, sendo 17 milhões em decorrência de DCV. ${ }^{4}$

No Brasil, em 2016, as DCNT resultaram em 707 mil óbitos, dos quais 362 mil foram por DCV. ${ }^{5}$ Em virtude da dimensão continental do país, a dinâmica da mortalidade por DCNT entre as regiões e unidades da federação brasileira não ocorre de modo homogêneo. São Paulo, por exemplo, segue o padrão dos países desenvolvidos, com redução da taxa de mortalidade, ${ }^{6}$ enquanto na região Nordeste, sob contexto socioeconômico diferente (piores indicadores socioeconômicos e difícil acesso à saúde), o cenário é o oposto. ${ }^{7,8}$

Dentre as DCV, as mais prevalentes são as doenças isquêmicas do coração (DIC), responsáveis por 116 mil óbitos no Brasil somente no ano de 2016. ${ }^{5}$ As DIC apresentam-se como um fluxo sanguíneo insuficiente e suprimento de oxigênio inadequado ao coração, cujas consequências microscópicas serão: lesão isquêmica do miocárdio, dano irreversível aos cardiomiócitos e hipertrofia de sobrecarga em áreas não necróticas. ${ }^{9}$ De forma pragmática, os indivíduos apresentam aumento na dependência das atividades de vida diária e de mobilidade, ou seja, há diminuição da capacidade funcional. ${ }^{10}$

Os fatores de risco dessas doenças são classificados em duas categorias: modificáveis e não modificáveis. Os fatores modificáveis são hipertensão arterial sistêmica (HAS), obesidade, sedentarismo, hábitos alimentares inadequados, tabagismo, consumo de bebidas alcoólicas, dislipidemias e resistência à insulina; os não modificáveis referem-se a idade, gênero, raça e hereditariedade..$^{11,12} \mathrm{O}$ controle dos fatores de risco modificáveis reduz em larga escala a morbimortalidade pelas doenças cardiovasculares. ${ }^{13}$

A realização de investigações sobre a evolução temporal da mortalidade por DCV no Nordeste é fundamental para a tomada de decisão em saúde, já que podem contribuir para a definição de áreas prioritárias de intervenção e para o desenvolvimento de estratégias e ações voltadas para a melhoria de saúde da população, sobretudo no que diz respeito aos fatores de risco. ${ }^{14}$
A região Nordeste carece de estudos descritivos pormenorizados sobre suas características epidemiológicas. Com base no exposto, o presente estudo objetivou descrever o perfil sociodemográfico e analisar tendência da taxa de mortalidade por DIC, segundo sexo e por faixa etária, nos estados da região Nordeste do Brasil, de 1996 a 2016. Dessa forma, ampliase o leque científico acerca de sua situação em saúde e os determinantes sociais que a compõem.

\section{Métodos}

\section{Desenho de estudo e fonte de dados}

Trata-se de estudo de séries temporais envolvendo todos os óbitos por DIC ocorridos no Nordeste brasileiro, no período de 1996 a 2016. Os registros dos óbitos foram obtidos do Sistema de Informações sobre Mortalidade (SIM) do Departamento de Informática do Sistema Único de Saúde do Ministério da Saúde (http://www.datasus.gov.br/). No processo de coleta, foram considerados os códigos I20 a I25 da Classificação Internacional de Doenças (CID-10): 120 - angina pectoris; I21 - infarto agudo do miocárdio; I22 - infarto do miocárdio recorrente; I23 - algumas complicações atuais subsequentes ao infarto agudo do miocárdio; 124 - outras doenças isquêmicas agudas do coração; 125 - doença isquêmica crônica do coração. Os dados populacionais necessários para o cálculo dos indicadores foram coletados do IBGE, censo de 2010.

\section{Área de estudo}

A região Nordeste do Brasil é composta por nove estados (Maranhão, Piauí, Ceará, Rio Grande do Norte, Paraíba, Pernambuco, Alagoas, Sergipe e Bahia) e uma população estimada de 57 milhões de habitantes, o que corresponde a $28 \%$ da população nacional - segunda região mais populosa do Brasil. ${ }^{15}$

\section{Variáveis de estudo}

Foram incluídas no estudo variáveis sociodemográficas (faixa etária, escolaridade, estado civil, cor/raça e unidades da federação), categoria da classificação internacional de doenças (CID-10) e as taxas brutas e padronizadas de mortalidade, segundo gênero e faixa etária.

Para o cálculo das taxas, foram utilizadas as seguintes equações: 
a) Taxa de mortalidade anual = (número de óbitos por DIC no local e ano / População residente no local e ano) x 100 mil

b) Taxa de mortalidade do perído = (Média simples do número de óbitos por DIC, do período, no local / População residente no meio do período no local) x 100 mil

Para a padronização das taxas de mortalidade, adotou-se o método direto, considerando como população padrão a brasileira do ano de 2010. Foram adotadas as seguintes faixas etárias no processo de padronização: 0 a 9 anos, 10 a 19 anos, 20 a 29 anos, 30 a 39 anos, 40 a 49 anos, 50 a 59 anos e 60 anos ou mais anos.

\section{Tratamento estatístico}

Inicialmente, as variáveis sociodemográficas foram analisadas com o emprego da estatística descritiva simples (frequências absoluta e relativa). A análise temporal foi realizada com o emprego do modelo de regressão por pontos de inflexão (joinpoint regression model). O modelo analisa se uma linha com múltiplos segmentos é mais adequada para explicar o comportamento temporal de um conjunto de dados quando comparada com uma linha reta ou com menos segmentos. Dessa forma, a tendência de cada indicador é classificada em estacionária, crescente ou decrescente, conforme inclinação da reta de regressão. Foram calculadas a variação percentual anual (APC, Annual Percent Change) e a variação média do período (AAPC, Average Annual Percent Change). ${ }^{16}$

$\mathrm{Na}$ análise, foram adotados os seguintes parâmetros: i) mínimo de zero joins, ii) máximo de quatro joins, iii) seleção do modelo pelo teste de computação de Monte Carlo $(n=$ 4.499 permutações), iv) método de autocorrelação dos erros baseado na data, v) intervalo de confiança de 95\% (IC95\%) e vi) nível de significância de $5 \%$. Tais análises foram realizadas com o auxílio do Joinpoint regression program (version 4.5.0.1, National Cancer Institute, Bethesda, MD, USA).

\section{Aspectos éticos}

O presente estudo utilizou dados secundários de domínio público, nos quais não é possível a identificação dos sujeitos, razão pela qual dispensou aprovação pelo comitê de ética em pesquisa.

\section{Resultados}

No período de 1996 a 2016, foram registrados 405.916 óbitos por DIC na região Nordeste do Brasil. Desses óbitos, $56,42 \%(n=229.006)$ eram homens; $74,25 \%(n=301.379)$, idosos; 48,76\% ( $n=197.936)$, pardos; 57,30\%, $(n=232.599)$ com ensino fundamental ou $<4$ anos na escola; e 44,25\% $(n=179.599)$, casados. Destacou-se a proporção de campos ignorados nas variáveis escolaridade $(33,54 \%)$, estado civil (8,95\%) e raça/cor (15,84\%). Quanto à causa do óbito, 85,07\% $(\mathrm{n}=345.329)$ decorreram de infarto agudo do miocárdio (I21); $46,37 \%(n=188.217)$ dos óbitos residiam nos estados de Pernambuco e da Bahia (Tabela 1).

A taxa de mortalidade regional padronizada passou de 30,7/100 mil habitantes em 1996 para 53,8/100 mil em 2016 (AAPC 2,8\%; IC95\%: 1,9 a 3,7; p<0,01). Todos os nove estados apresentaram tendência significativa de crescimento, destacando-se o estado do Maranhão, cuja taxa passou de 14,8/100 mil em 1996 para 64,0/100 mil habitantes em 2016 (AAPC 7,6\%; IC95\%: 5,7 a 9,6; p<0,01), seguido do Piauí, no qual a taxa ascendeu de 18,7/100 mil em 1996 para 61,5/100 mil habitantes em 2016 (AAPC 6,0\%; IC95\%: 4,3 a $7,8 ; p<0,01)$. Pernambuco destacou-se com maior número de segmentos temporais, com quatro inflexões, cinco segmentos temporais e AAPC igual a 1,2\% (IC95\%: 0,0 a 2,5; $\mathrm{p}<0,01$ ). Os estados do Rio Grande do Norte e de Alagoas apresentaram tendência linear de crescimento da mortalidade $(2,2 \%$ no Rio Grande do Norte e 3,6\% em Alagoas) (Figura 1).

A taxa de mortalidade média no sexo masculino $(42,05 / 100$ mil) foi $32 \%$ maior que no feminino $(31,6 / 100 \mathrm{mil})$, ainda que com o mesmo percentual de variação médio (AAPC 4,4\%; IC95\%: 3,4 a 5,4; p<0,01). A faixa etária de adolescentes apresentou o maior aumento percentual médio do período (AAPC: 5,2\%, p<0,01) (Tabela 2). Todos os estados apresentaram tendência de crescimento em ambos os sexos, destacando-se o Maranhão (AAPC 8,7\% para o masculino e 9,3\% para o feminino) e o Piauí (AAPC 7,9\% para o masculino e $8,4 \%$ para o feminino) (Tabela 3).

Na estratificação segundo faixa etária, o modelo de regressão apontou tendência de crescimento da mortalidade em todos os segmentos etários, com exceção do grupo com idade entre 30 e 39 anos (AAPC 0,9\%; IC95\%: -0,8 a 2,5; p=0,4) (Tabela 2). A taxa de mortalidade regional em indivíduos com 60 anos ou mais passou de 202,0/100 mil em 1996 para 382,3/100 mil habitantes em 2016, com crescimento médio anual de 3,3\% (IC95\%: 2,5 a 4,1; p<0,01). A faixa etária de 60 anos ou mais foi a única com crescimento estatisticamente significativo em todos os estados, destacando-se os estados do Maranhão (AAPC 8,4\%; IC95\%: 6,3 a 10,5; p<0,01) e do Piauí (AAPC 6,5\%; IC 95\%: 5,3 a 7,7; $\mathrm{p}<0,01$ ) (Tabela 4).

Por fim, observou-se divergência na tendência segundo faixa etária ao comparar os estados. No Piauí, por exemplo, houve crescimento em todas as faixas a partir dos 10 anos de idade; ao passo que, na Bahia, o crescimento foi observado apenas em idosos (60 anos ou mais) (Tabela 4).

\section{Discussão}

Este trabalho analisou o perfil sociodemográfico e o comportamento temporal da mortalidade por DIC na região Nordeste do Brasil no período de 1996 a 2016. O perfil dos óbitos caracterizou-se pelo predomínio do sexo masculino, acometimento de idosos, raça/cor parda e baixa escolaridade. A análise temporal demonstrou crescimento da taxa de mortalidade na região e em todos os estados, com destaque para o Maranhão e o Piauí.

O perfil observado neste estudo está em consonância com a literatura. ${ }^{1,7,17-20} \mathrm{O}$ processo de envelhecimento da população traz consigo uma ampliação dos fatores de risco para DCV, destacando-se as dislipidemias, a obesidade e a HAS. ${ }^{2}$ Estudo realizado no município de São Paulo aponta que a razão de chance de desenvolver DCV é maior em diabéticos (odds ratio [OR] 1,90), tabagistas (OR 1,49), indivíduos com sobrepeso (OR 1,57) e hipertensos (OR 2,22). ${ }^{20}$ Estima-se que a prevalência de HAS em idosos seja seis a oito vezes superior à de adultos jovens, justificada pelo controle pressórico deficitário, em razão 


\begin{tabular}{|c|c|c|c|c|c|c|c|c|}
\hline \multirow[t]{2}{*}{ Variável } & \multicolumn{2}{|c|}{$\begin{array}{c}\text { Masculino } \\
\mathrm{n}=229006(56,42 \%)\end{array}$} & \multicolumn{2}{|c|}{$\begin{array}{c}\text { Feminino } \\
\mathrm{n}=176766(43,55 \%)\end{array}$} & \multicolumn{2}{|c|}{$\begin{array}{c}\text { Ignorado } \\
n=144(0,03 \%)\end{array}$} & \multicolumn{2}{|c|}{$\begin{array}{c}\text { Total } \\
\mathrm{n}=405916(100 \%) \\
\end{array}$} \\
\hline & $\mathrm{n}$ & $\%$ & $\mathrm{n}$ & $\%$ & $\mathbf{n}$ & $\%$ & n & $\%$ \\
\hline \multicolumn{9}{|l|}{ Faixa etária } \\
\hline$<10$ anos & 72 & 0,05 & 49 & 0,02 & 0 & 0,00 & 121 & 0,03 \\
\hline 10 a 19 & 469 & 0,20 & 215 & 0,12 & 1 & 0,70 & 685 & 0,17 \\
\hline 20 a 29 & 2197 & 0,96 & 815 & 0,46 & 0 & 0,00 & 3012 & 0,74 \\
\hline 30 a 39 & 7086 & 3,09 & 3197 & 1,81 & 3 & 2,08 & 10286 & 2,53 \\
\hline 40 a 49 & 19380 & 8,46 & 10763 & 6,09 & 14 & 9,72 & 30157 & 7,43 \\
\hline 50 a 59 & 38131 & 16,65 & 21493 & 12,16 & 17 & 11,81 & 59641 & 14,69 \\
\hline 60 anos ou mais & 161295 & 70,43 & 139991 & 79,20 & 93 & 64,58 & 301379 & 74,25 \\
\hline Ignorado & 376 & 0,16 & 243 & 0,14 & 16 & 11,11 & 635 & 0,16 \\
\hline \multicolumn{9}{|l|}{ Cor/raça } \\
\hline Branca & 58574 & 25,58 & 53120 & 30,05 & 10 & 6,94 & 111704 & 27,52 \\
\hline Preta & 17345 & 7,57 & 11975 & 6,77 & 1 & 0,70 & 29321 & 7,22 \\
\hline Amarela & 1062 & 0,46 & 928 & 0,53 & 2 & 1,39 & 1992 & 0,49 \\
\hline Parda & 115714 & 50,53 & 82208 & 46,51 & 14 & 9,72 & 197936 & 48,76 \\
\hline Indígena & 385 & 0,17 & 301 & 0,17 & 0 & 0,00 & 686 & 0,17 \\
\hline Ignorado & 35926 & 15,69 & 28234 & 15,97 & 117 & 81,25 & 64277 & 15,84 \\
\hline \multicolumn{9}{|l|}{ Escolaridade } \\
\hline Fundamental ou $<4$ anos na escola & 129138 & 56,39 & 103426 & 58,51 & 35 & 24,30 & 232599 & 57,30 \\
\hline Médio & 15866 & 6,93 & 9088 & 5,14 & 4 & 2,78 & 24958 & 6,15 \\
\hline Superior & 8303 & 3,62 & 3909 & 2,21 & 0 & 0,00 & 12212 & 3,01 \\
\hline Ignorado & 75699 & 33,06 & 60343 & 34,14 & 105 & 72,92 & 136147 & 33,54 \\
\hline \multicolumn{9}{|l|}{ Estado civil } \\
\hline Solteiro & 45124 & 19,7 & 41989 & 23,75 & 21 & 14,58 & 87134 & 21,47 \\
\hline Casado & 125600 & 54,85 & 53953 & 30,52 & 46 & 31,94 & 179599 & 44,25 \\
\hline Viúvo & 26413 & 11,53 & 58356 & 33,01 & 26 & 18,06 & 84795 & 20,89 \\
\hline Separado judicialmente & 6930 & 3,03 & 3642 & 2,06 & 1 & 0,70 & 10573 & 2,60 \\
\hline Outro & 5254 & 2,29 & 2228 & 1,27 & 2 & 1,39 & 7484 & 1,84 \\
\hline Ignorado & 19685 & 8,60 & 16598 & 9,39 & 48 & 33,33 & 36331 & 8,95 \\
\hline \multicolumn{9}{|l|}{ Categoria CID-10 } \\
\hline 120 & 885 & 0,39 & 875 & 0,5 & 0 & 0,00 & 1760 & 0,43 \\
\hline 121 & 196621 & 85,86 & 148585 & 84,06 & 123 & 85,42 & 345329 & 85,07 \\
\hline 122 & 591 & 0,25 & 386 & 0,21 & 0 & 0,00 & 977 & 0,25 \\
\hline 123 & 3 & $<0,01$ & 0 & 0,00 & 0 & 0,00 & 3 & $<0,01$ \\
\hline 124 & 6745 & 2,95 & 6320 & 3,58 & 7 & 4,86 & 13072 & 3,22 \\
\hline 125 & 24161 & 10,55 & 20600 & 11,65 & 14 & 9,72 & 44775 & 11,03 \\
\hline \multicolumn{9}{|l|}{ Estados } \\
\hline Maranhão & 21847 & 9,54 & 13792 & 7,8 & 24 & 16,67 & 35663 & 8,79 \\
\hline Piauí & 14539 & 6,35 & 9446 & 5,34 & 16 & 11,11 & 24001 & 5,91 \\
\hline Ceará & 33500 & 14,63 & 26623 & 15,06 & 26 & 18,06 & 60149 & 14,82 \\
\hline Rio Grande do Norte & 17347 & 7,57 & 13335 & 7,54 & 4 & 2,78 & 30686 & 7,56 \\
\hline Paraíba & 18825 & 8,22 & 14945 & 8,45 & 11 & 7,64 & 33781 & 8,32 \\
\hline Pernambuco & 59385 & 25,93 & 48909 & 27,67 & 34 & 23,61 & 108328 & 26,69 \\
\hline Alagoas & 11957 & 5,22 & 9034 & 5,11 & 2 & 1,38 & 20993 & 5,17 \\
\hline Sergipe & 6797 & 2,97 & 5624 & 3,20 & 5 & 3,47 & 12426 & 3,06 \\
\hline Bahia & 44809 & 19,57 & 35058 & 19,83 & 22 & 15,28 & 79889 & 19,68 \\
\hline
\end{tabular}



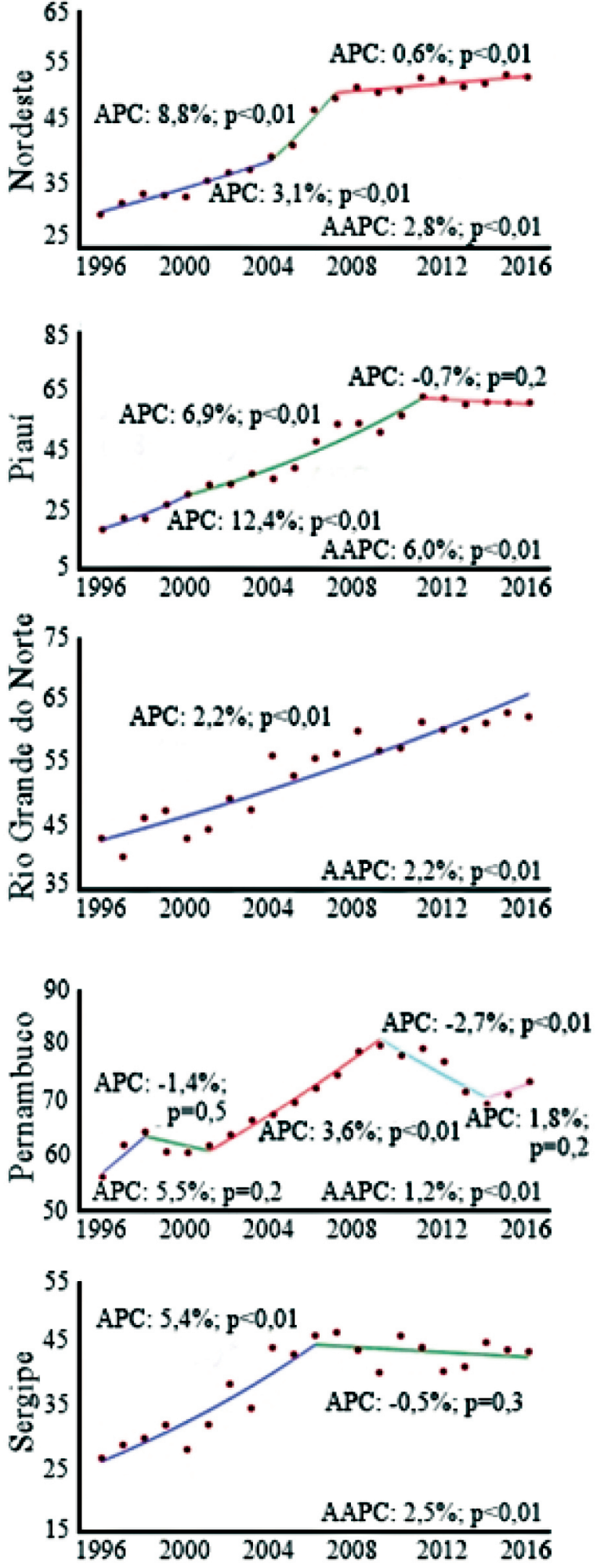
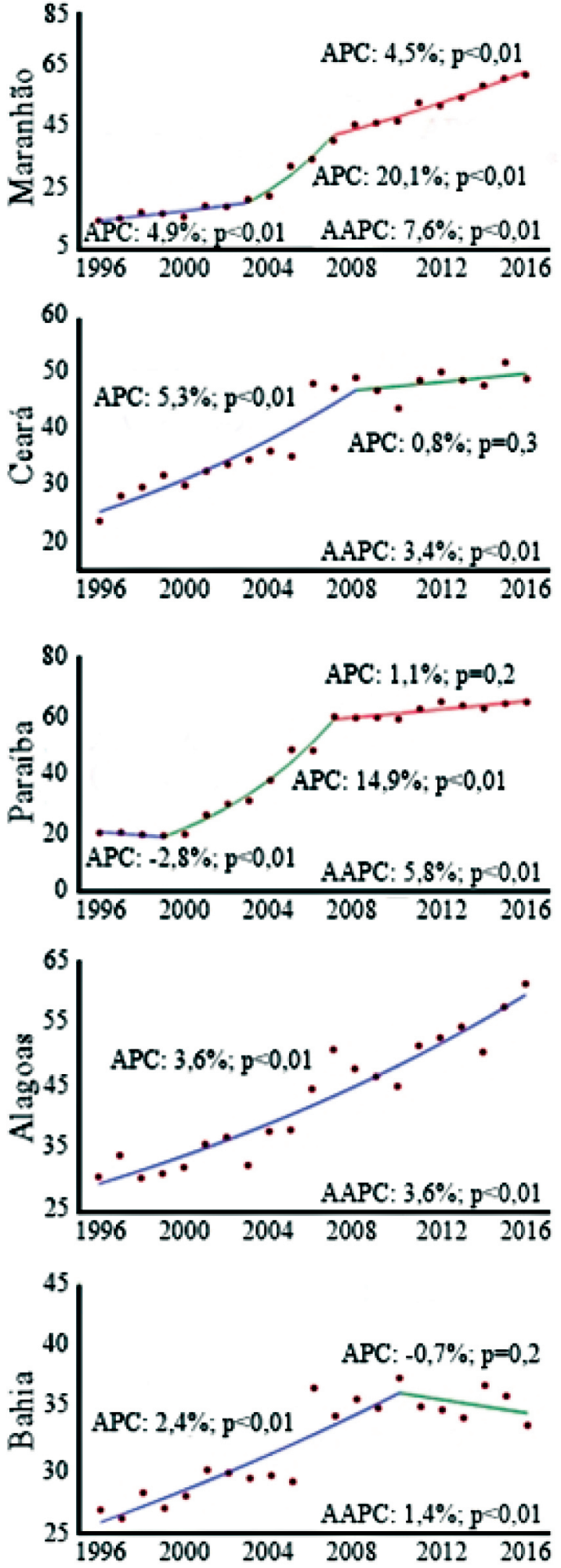

Figura 1 - Tendência temporal da taxa de mortalidade padronizada por doenças isquêmicas do coração (DIC), segundo estado de residência. Nordeste, Brasil, 1996-2016. APC: Annual Percent Change. AAPC: Average Annual Percent Change. * Valores de intervalo de confiança foram suprimidos da ilustração para melhor visualização e entendimento das linhas de tendência. 


\begin{tabular}{|c|c|c|c|c|c|c|}
\hline \multirow{2}{*}{ Variável } & \multicolumn{3}{|c|}{ Taxa de mortalidade/100 mil } & \multicolumn{3}{|c|}{ Tendência } \\
\hline & 1996 & 2016 & $1996-2016$ & Período & APC (IC95\%; p valor) & AAPC (IC95\%; p valor) \\
\hline \multicolumn{7}{|l|}{ Sexo } \\
\hline \multirow{3}{*}{ Masculino } & \multirow{3}{*}{25,52} & \multirow{3}{*}{60,88} & \multirow{3}{*}{42,05} & 1996-2004 & $3,7 \%(3,0$ a 4,$5 ; p<0,01)$ & \multirow{3}{*}{$4,4 \%$ (3,4 a 5,$4 ; p<0,01)$} \\
\hline & & & & $2004-2007$ & $10,6 \%(3,4$ a 18,$3 ; p<0,01)$ & \\
\hline & & & & $2007-2016$ & $3,0 \%(2,4$ a 3,$6 ; p<0,01)$ & \\
\hline \multirow{3}{*}{ Feminino } & \multirow{3}{*}{18,78} & \multirow{3}{*}{44,24} & \multirow{3}{*}{31,69} & 1996-2003 & $4,0 \%(3,1$ a 5,$0 ; p<0,01)$ & \multirow{3}{*}{$4,4 \%(3,6$ a 5,$2 ; p<0,01)$} \\
\hline & & & & $2003-2007$ & $9,2 \%(5,5$ a 13,$1 ; p<0,01)$ & \\
\hline & & & & $2007-2016$ & $2,6 \%(2,0$ a 3,$2 ; p<0,01)$ & \\
\hline \multicolumn{7}{|l|}{ Faixa Etária } \\
\hline 0 a 9 & 0,01 & 0,10 & 0,05 & - & - & - \\
\hline 10 a 19 & 0,21 & 0,39 & 0,31 & $1996-2016$ & $5,2 \%(3,0$ a 7,$5 ; p<0,01)$ & $5,2 \%(3,0$ a 7,$5 ; p<0,01)$ \\
\hline 20 a 29 & 1,00 & 1,96 & 1,42 & $1996-2016$ & $2,8 \%(2,0$ a 3,$6 ; p<0,01)$ & $2,8 \%(2,0$ a 3,$6 ; p<0,01)$ \\
\hline \multirow{3}{*}{30 a 39} & \multirow{3}{*}{5,79} & \multirow{3}{*}{7,18} & \multirow{3}{*}{6,66} & 1996-2003 & $-1,5 \%(-3,5$ a 0,$5 ; p=0,2)$ & \multirow{3}{*}{$0,9 \%(-0,8$ a 2,$5 ; p=0,4)$} \\
\hline & & & & $2003-2007$ & $6,8 \%(-1,1$ a 15,$4 ; p=0,3)$ & \\
\hline & & & & $2007-2016$ & $0,2 \%(-1,2$ a 1,$6 ; p=0,2)$ & \\
\hline \multirow{4}{*}{40 a 49} & \multirow{4}{*}{20,44} & \multirow{4}{*}{26,84} & \multirow{4}{*}{25,38} & 1996-1998 & $9,8 \%(1,1$ a 19,$2 ; p<0,01)$ & \multirow{4}{*}{$1,5 \%(0,1$ a 2,$9 ; p<0,01)$} \\
\hline & & & & 1998-2001 & $-4,6 \%(-12,1$ a 3,6; $p=0,2)$ & \\
\hline & & & & $2001-2008$ & $4,4 \%(3,0$ a 5,$9 ; p<0,01)$ & \\
\hline & & & & $2008-2016$ & $-0,7 \%(-1,6$ a 0,$2 ; p=0,2)$ & \\
\hline \multirow{2}{*}{50 a 59} & \multirow{2}{*}{52,99} & \multirow{2}{*}{78,25} & \multirow{2}{*}{74,40} & 1996-2008 & $3,3 \%(2,7$ a 3,$9 ; p<0,01)$ & \multirow{2}{*}{$1,6 \%(1,1$ a 2,$2 ; p<0,01)$} \\
\hline & & & & $2008-2016$ & $-0,7 \%(-1,8$ a 0,$3 ; p=0,3)$ & \\
\hline \multirow{3}{*}{60 ou mais } & \multirow{3}{*}{202,03} & & & $1996-2003$ & $3,3 \%(2,2$ a 4,$3 ; p<0,01)$ & \\
\hline & & 382,28 & 323,15 & 2003-2007 & $8,5 \%(4,6$ a 12,$6 ; p<0,01)$ & $3,3 \%(2,5$ a 4,$1 ; p<0,01)$ \\
\hline & & & & $2007-2016$ & $1,0 \%(0,4$ a 1,$7 ; p<0,01)$ & \\
\hline & & & & 1996-2004 & $4,0 \%(3,3$ a 4,$7 ; p<0,01)$ & \\
\hline População geral & 22,14 & 52,40 & 36,82 & $2004-2007$ & $10,5 \%(3,9$ a 17,$4 ; p<0,01)$ & $4,4 \%(3,5$ a 5,$3 ; p<0,01)$ \\
\hline & & & & $2007-2016$ & $2,8 \%(2,2$ a 3,$4 ; p<0,01)$ & \\
\hline
\end{tabular}

APC: Annual Percent Change. AAPC: Average Annual Percent Change.

da cronicidade da doença e sensibilidade e restrições na terapia farmacológica. Em estudo realizado em Goiânia/GO, 912 indivíduos com HAS foram entrevistados, 72,6\% estavam em tratamento e apenas 50,8\% apresentavam controle pressórico. ${ }^{21}$

É pertinente destacar o crescimento da mortalidade na adolescência, conforme observado nesta investigação. Nessa população, estudos apontam um amplo conjunto de fatores associados ao sobrepeso e obesidade, tais como i) ingestão excessiva de açúcares simples e gorduras, ii) consumo insuficiente de frutas e hortaliças e iii) sedentarismo, que podem resultar em mortalidade precoce. ${ }^{22} \mathrm{O}$ Estudo de Riscos Cardiovasculares em Adolescentes (ERICA), de 2013 a 2014, relatou prevalência de síndrome metabólica em 3,3\% dos que estavam em sobrepeso e em $21,7 \%$ dos que apresentavam obesidade no Nordeste. ${ }^{23}$

A importância médica de alterações no perfil lipídico está relacionada à presença de aterosclerose subclínica e à possibilidade de predizer dislipidemia em vida adulta. No
ERICA, observou-se maior prevalência de baixos níveis de lipoproteína de alta densidade (HDL, high density lipoproteins) nas regiões Norte e Nordeste do Brasil. ${ }^{24}$ Estudo realizado em Belém/PA em 2006, envolvendo 437 crianças e adolescentes, apontou $28,8 \%$ com excesso de peso e $36 \%$ com percentual de gordura elevado. Destas, 49\% apresentaram alteração no perfil lipídico, com destaque para os baixos valores de HDL. ${ }^{25}$ Resultados semelhantes foram observados em Porto Alegre/RS, onde ocorre um crescimento da dislipidemia secundária à obesidade, caracterizada por baixos níveis de HDL e aumento dos níveis de triglicerídeos, secundário à resistência à insulina. ${ }^{26}$

O risco cardiovascular também recebe influência do sexo, sendo maior na população masculina. Um estudo sobre hipertensão em Goiânia/GO, realizado em 2010, mostrou que a taxa de controle dos níveis pressóricos é menor na população masculina (44,0\% em homens e 54,8\% em mulheres). ${ }^{21}$ Em 
Montes Claros/MG, 62,8\% dos casos de DM são mulheres, justificados pela maior procura por assistência médica por essa população, que apresenta maior e melhor adesão ao tratamento. ${ }^{27}$ Ademais, as mulheres tem características biológicas e comportamentais capazes de reduzir o risco de doenças cardiovasculares, destacando-se o papel protetor do estradiol no endotélio vascular, o maior acesso aos serviços de saúde e o melhor desempenho no controle de fatores risco. ${ }^{17}$

A influência do desenvolvimento socioeconômico e do acesso aos serviços de saúde no padrão de mortalidade tem sido evidenciada em diferentes estudos. ${ }^{28-30}$ Investigação realizada em 2012, nos estados do Rio de Janeiro, Rio Grande do Sul e São Paulo, mostrou correlação entre aumento do PIB per capita e do nível de escolaridade com a diminuição da mortalidade por DIC. ${ }^{31}$ No Nordeste, o Maranhão, ao mesmo tempo em que apresentou o maior crescimento percentual médio $(7,6 \%)$ na mortalidade por DIC, caracteriza-se por ser o estado com o menor PIB per capita - $\mathrm{R} \$ 400,97$ - e o segundo estado com maior taxa de analfabetismo entre pessoas com 18 anos de idade ou mais $(20,56 \%)$, atrás somente de Alagoas $(21,47 \%)^{32}$

No Rio Grande do Sul, fora evidenciada correlação positiva para índice GINI, escolaridade e distância geográfica $-p=0,001$ - com a taxa de mortalidade por DIC. A distância geográfica mostra que as distribuições espaciais de centros de referência em intervenção cardiológica influenciam diretamente como fator preditor de óbito, independente, na taxa de mortalidade. ${ }^{33}$ Outro estudo relata ainda que, em consonância às disparidades socioeconômicas, o fator distância geográfica torna-se ainda mais grave. Não obstante, a criação de novos serviços de referência em cardiologia é uma possibilidade para a solução do problema. ${ }^{34}$

O Brasil tem empreendido esforços no sentido de reduzir a ocorrência de DIC. Em 2000, o Ministério da Saúde implantou o Plano de Reorganização da Atenção à Hipertensão Arterial e ao Diabetes Melito. Seus objetivos visam dispor de um sistema informatizado que auxilia no cadastramento e acompanhamento dos portadores de HAS e DM - HiperDia, bem como conhecer a magnitude das doenças, planejar a aquisição de medicamentos e capacitar os profissionais na rede de saúde brasileira para atuar no perfil de atenção à saúde do sistema único de saúde (SUS). ${ }^{35}$

Neste âmbito, estudo realizado em Maringá/PR demonstrou que o impacto das políticas públicas, como a Estratégia de Saúde da Família (ESF), está associado às menores taxas de internação por condições cardiovasculares sensíveis à atenção primária (CCSAP). O sucesso da atenção primária à saúde (APS) é embasado por sua abordagem sobre os fatores de risco para DCV, no suporte ao autogerenciamento da saúde junto ao acompanhamento longitudinal por equipes de saúde, além do empoderamento e da autonomia. ${ }^{36}$

A APS contribui no combate ao tabagismo, na ampliação do acesso aos serviços de saúde e na distribuição de medicamentos para controle dos fatores de risco da DIC. ${ }^{37} \mathrm{Em}$ uma pesquisa sobre acesso aos medicamentos pelos usuários da APS, a dimensão de disponibilidade de medicamentos estava baixa, em torno de $46,3 \%$ a $64,3 \%$, bem menos que os $80 \%$ preconizados pela Organização Mundial da Saúde (OMS). ${ }^{38}$ A alternativa proposta é substituir o medicamento em falta e encaminhar para a Farmácia Popular, embora o controle de doenças crônicas possa vir a ser prejudicado por essa medida. ${ }^{39}$
A tendência de crescimento da mortalidade não ocorre de modo homogêneo entre os estados nordestinos: há crescimento linear em Alagoas e no Rio Grande do Norte, instabilidade das taxas em Pernambuco e um padrão estacionário na maioria dos estados a partir da segunda metade da primeira década do século XXI. Diferentes fatores podem justificar esses achados, tais como as diferenças socioeconômicas intrarregionais, a influência das políticas públicas e a qualidade dos registros de informações. ${ }^{3,40-43}$

Mesmo considerando os cuidados metodológicos adotados, este estudo tem limitações, com destaque para a qualidade dos registros de mortalidade, sendo este um desafio para o adequado monitoramento da situação de saúde e auxílio na tomada de decisão. Adicionalmente, a existência de código garbage e as dificuldades operacionais na vigilância do óbito são fatores adicionais que comprometem a qualidade dos registros.

\section{Conclusão}

As taxas de mortalidade na região Nordeste do Brasil apresentaram crescimento significativo em todos os estados que a compõem, sendo maior no Maranhão e no Piauí. O perfil de óbitos caracterizou-se pelo predomínio de homens idosos, raça/cor parda, ensino fundamental ou $<4$ anos na escola e casados. Durante o período, o sexo masculino demonstrou taxas significativamente maiores que o sexo feminino, ainda que com o mesmo crescimento percentual. As desigualdades nas taxas entre os estados demonstram a necessidade de estratégias consoantes com a realidade e as particularidades locais, e a possível influência das condições de vida da população, sendo essa uma recomendação para estudos futuros. Considerando o achado preocupante de maior taxa de incremento anual na faixa etária de adolescentes, são necessárias maiores investigações sobre a mortalidade cardiovascular nos mesmos.

\section{Contribuição dos autores}

Concepção e desenho da pesquisa e Obtenção de dados: Santana GBA, Oliveira TF, Mesquita RR, Gomes JA, Souza CDF, Rodrigues AKBF; Análise e interpretação dos dados: Santana GBA, Leal TC, Paiva JPS, Silva LF, Santos LG, Gomes JA, Souza CDF, Rodrigues AKBF; Análise estatística: Santana GBA, Leal TC, Paiva JPS, Silva LF, Santos LG, Souza CDF, Rodrigues AKBF; Redação do manuscrito: Santana GBA, Leal TC, Paiva JPS, Silva LF, Oliveira TF, Gomes JA, Souza CDF, Rodrigues AKBF; Revisão crítica do manuscrito quanto ao conteúdo intelectual importante: Santana GBA, Leal TC, Paiva JPS, Silva LF, Santos LG, Souza CDF, Rodrigues AKBF.

\section{Potencial conflito de interesse}

Não há conflito com o presente artigo

\section{Fontes de financiamento}

O presente estudo não teve fontes de financiamento externas.

\section{Vinculação acadêmica}

Não há vinculação deste estudo a programas de pósgraduação. 
Tabela 3 - Percentual de variação médio (AAPC) da taxa de mortalidade por doenças isquêmicas do coração (DIC), segundo sexo e estado de residência. Nordeste, Brasil, 1996-2016

\begin{tabular}{|c|c|c|c|c|c|c|}
\hline \multirow{2}{*}{ Estado } & \multicolumn{3}{|c|}{ Masculino } & \multicolumn{3}{|c|}{ Feminino } \\
\hline & 1996 & 2016 & AAPC (IC95\%; p valor) & 1996 & 2016 & AAPC (IC95\%; p valor) \\
\hline Maranhão & 12,06 & 62,75 & $8,7 \%(7,0$ a 10,$5 ; p<0,01)$ & 6,20 & 38,89 & $9,3 \%(7,1$ a 11,$7 ; p<0,01)$ \\
\hline Piauí & 16,16 & 75,49 & $7,9 \%(4,4$ a 11,$5 ; p<0,01)$ & 9,98 & 45,16 & $8,4 \%(7,3$ a 9,$5 ; p<0,01)$ \\
\hline Ceará & 20,65 & 56,89 & $5,1 \%(4,5$ a 5,$8 ; p<0,01)$ & 15,55 & 41,70 & $5,0 \%(3,9$ a 5,$1 ; p<0,01)$ \\
\hline Rio Grande do Norte & 39,61 & 74,56 & $3,5 \%(3,1$ a 3,$9 ; p<0,01)$ & 27,88 & 53,24 & $3,9 \%(3,4$ a 4,$4 ; p<0,01)$ \\
\hline Paraíba & 20,88 & 78,08 & $6,8 \%(5,5$ a 8,$1 ; p<0,01)$ & 15,53 & 60,00 & $7,1 \%(5,2$ a 9,$1 ; p<0,01)$ \\
\hline Pernambuco & 49,59 & 85,82 & $2,4 \%(1,5$ a 3,$3 ; p<0,01)$ & 36,93 & 64,36 & $2,3 \%(1,3$ a 3,$3 ; p<0,01)$ \\
\hline Alagoas & 23,73 & 61,02 & $4,7 \%(3,1$ a 6,$3 ; p<0,01)$ & 15,95 & 47,96 & $5,3 \%(4,5$ a 6,$1 ; p<0,01)$ \\
\hline Sergipe & 19,25 & 42,47 & $4,4 \%(3,1$ a 5,$7 ; p<0,01)$ & 18,58 & 35,80 & $2,7 \%(0,5$ a 4,$9 ; p<0,01)$ \\
\hline Bahia & 21,35 & 39,39 & $3,6 \%(3,2$ a 4,$1 ; p<0,01)$ & 16,32 & 29,05 & $3,6 \%(2,7$ a 4,$4 ; p<0,01)$ \\
\hline
\end{tabular}

APC: Annual Percent Change. AAPC: Average Annual Percent Change.

Tabela 4 - Percentual de variação médio (AAPC) da taxa de mortalidade bruta por doenças isquêmicas do coração (DIC), segundo faixa etária e estado de residência. Nordeste, Brasil, 1996-2016

\begin{tabular}{|c|c|c|c|c|c|c|c|c|c|}
\hline \multirow{3}{*}{$\begin{array}{l}\text { Faixa } \\
\text { etária }\end{array}$} & \multicolumn{3}{|r|}{ Maranhão } & \multicolumn{3}{|r|}{ Piauí } & \multicolumn{3}{|r|}{ Ceará } \\
\hline & \multicolumn{2}{|c|}{ Taxas } & \multirow{2}{*}{$\begin{array}{c}\text { AAPC } \\
\text { (IC95\%) }\end{array}$} & \multicolumn{2}{|c|}{ Taxas } & \multirow{2}{*}{$\begin{array}{c}\text { AAPC } \\
\text { (IC95\%) }\end{array}$} & \multicolumn{2}{|c|}{ Taxas } & \multirow{2}{*}{$\begin{array}{c}\text { AAPC } \\
\text { (IC95\%) }\end{array}$} \\
\hline & 1996 & 2016 & & 1996 & 2016 & & 1996 & 2016 & \\
\hline 0 a 9 & 0,00 & 0,14 & - & 0,00 & 0,19 & - & 0,00 & 0,07 & - \\
\hline 10 a 19 & 0,00 & 0,50 & - & 0,15 & 0,84 & $7,0 \%(2,9$ a 11,$3 ; p<0,01)$ & 0,51 & 0,56 & $4,9 \%(-0,3$ a 10,$4 ; p=0,5)$ \\
\hline 20 a 29 & 0,12 & 3,25 & $9,0 \%(4,9$ a 13,$2 ; p<0,01)$ & 0,93 & 3,42 & $6,2 \%(3,6$ a 8,$9 ; p<0,01)$ & 1,16 & 1,39 & $0,7 \%(-0,6$ a 2,$1 ; p=0,3)$ \\
\hline 30 a 39 & 3,17 & 11,62 & $5,8 \%(4,7$ a 7,$0 ; p<0,01)$ & 6,09 & 10,00 & $3,5 \%(2,0$ a 5,$1 ; p<0,01)$ & 5,80 & 5,31 & $0,8 \%(-0,3$ a 1,$9 ; p=0,3)$ \\
\hline 40 a 49 & 10,46 & 35,96 & $6,1 \%(1,5$ a 11,$0 ; p<0,01)$ & 14,71 & 31,10 & $3,5 \%(2,5$ a 4,$5 ; p<0,01)$ & 15,29 & 20,40 & $2,1 \%(1,2$ a 3,$1 ; p<0,01)$ \\
\hline 50 a 59 & 29,12 & 90,17 & $5,7 \%(3,2$ a 8,$1 ; p<0,01)$ & 34,61 & 87,61 & $3,5 \%(1,4$ a 5,$6 ; p<0,01)$ & 38,07 & 66,06 & $1,9 \%(0,5$ a 3,$4 ; p<0,01)$ \\
\hline $60+$ & 94,19 & 446,37 & $8,4 \%(6,3$ a 10,$5 ; p<0,01)$ & 114,40 & 432,41 & $6,5 \%(5,3$ a 7,$7 ; p<0,01)$ & 157,12 & 361,26 & $3,9 \%(2,8$ a 5,$1 ; p<0,01)$ \\
\hline \multirow{3}{*}{$\begin{array}{l}\text { Faixa } \\
\text { etária }\end{array}$} & \multicolumn{3}{|c|}{ Rio Grande do Norte } & \multicolumn{3}{|r|}{ Paraíba } & \multicolumn{3}{|c|}{ Pernambuco } \\
\hline & \multicolumn{2}{|c|}{ Taxas } & AAPC & \multicolumn{2}{|c|}{ Taxas } & AAPC & \multicolumn{2}{|c|}{ Taxas } & AAPC \\
\hline & 1996 & 2016 & (IC95\%) & 1996 & 2016 & (IC95\%) & 1996 & 2016 & (IC95\%) \\
\hline 0 a 9 & 0,18 & 0,00 & - & 0,00 & 0,16 & - & 0,00 & 0,07 & - \\
\hline 10 a 19 & 0,34 & 0,34 & - & 0,51 & 0,15 & - & 0,23 & 0,54 & $4,5 \%(0,9$ a 8,$3 ; p<0,01)$ \\
\hline 20 a 29 & 1,14 & 1,83 & $4,7 \%(0,8$ a 8,$7 ; p<0,01)$ & 0,92 & 2,42 & $5,1 \%(2,7$ a 7,$5 ; p<0,01)$ & 2,10 & 2,75 & $1,5 \%(-0,3$ a 3,$2 ; p=0,4)$ \\
\hline 30 a 39 & 7,16 & 7,45 & $2,0 \%(0,2$ a 3,$7 ; p<0,01)$ & 4,12 & 7,30 & $4,7 \%(3,5$ a 5,$9 ; p<0,01)$ & 9,65 & 9,13 & $-1,1 \%(-5,9$ a 4,$1 ; p=0,4)$ \\
\hline 40 a 49 & 23,52 & 28,02 & $1,1 \%(0,2$ a 2,$0 ; p<0,01)$ & 14,54 & 34,14 & $4,2 \%(1,4$ a 7,$1 ; p<0,01)$ & 33,08 & 35,25 & $-0,2 \%(-3,3$ a 3,$0 ; p=0,2)$ \\
\hline 50 a 59 & 69,14 & 88,46 & $1,3 \%(0,4$ a 2,$2 ; p<0,01)$ & 31,84 & 84,49 & $5,7 \%(3,9$ a 7,$4 ; p<0,01)$ & 98,89 & 114,28 & $0,6 \%(-0,8$ a 2,$0 ; p=0,2)$ \\
\hline $60+$ & 298,31 & 452,97 & $2,4 \%(1,8$ a 3,$0 ; p<0,01)$ & 138,19 & 469,44 & $6,3 \%(4,8$ a 7,$7 ; p<0,01)$ & 378,85 & 520,30 & $1,2 \%(0,4$ a 1,$9 ; p<0,01)$ \\
\hline \multirow{3}{*}{$\begin{array}{l}\text { Faixa } \\
\text { etária }\end{array}$} & \multicolumn{3}{|r|}{ Alagoas } & \multicolumn{3}{|r|}{ Sergipe } & \multicolumn{3}{|r|}{ Bahia } \\
\hline & \multicolumn{2}{|c|}{ Taxas } & AAPC & \multicolumn{2}{|c|}{ Taxas } & AAPC & \multicolumn{2}{|c|}{ Taxas } & AAPC \\
\hline & 1996 & 2016 & (IC95\%) & 1996 & 2016 & (IC95\%) & 1996 & 2016 & (IC95\%) \\
\hline 0 a 9 & 0,00 & 0,17 & - & 0,00 & 0,00 & - & 0,00 & 0,08 & - \\
\hline 10 a 19 & 0,00 & 0,15 & - & 0,00 & 0,00 & - & 0,13 & 0,23 & - \\
\hline 20 a 29 & 0,66 & 2,13 & $3,2 \%(0,7$ a 5,$7 ; p<0,01)$ & 0,35 & 0,50 & $-2,9 \%(-7,1$ a 1,$5 ; p=0,4)$ & 0,75 & 0,99 & $0,8 \%(-0,7$ a 2,$3 ; p=0,6)$ \\
\hline 30 a 39 & 6,73 & 5,34 & $-0,8 \%(-2,3$ a 0,$6 ; p=0,2)$ & 5,59 & 4,21 & $1,1 \%(-0,9$ a 3,$1 ; p=0,3)$ & 4,31 & 5,34 & $0,4 \%(-0,5$ a 1,$2 ; p=0,6)$ \\
\hline 40 a 49 & 31,05 & 36,91 & $0,3 \%(-2,2$ a 2,$9 ; p=0,2)$ & 12,47 & 26,10 & $2,9 \%(-0,3$ a 6,$1 ; p=0,3)$ & 20,10 & 16,87 & $-0,1 \%(-0,8$ a 0,$6 ; p=0,7)$ \\
\hline 50 a 59 & 61,26 & 95,11 & $1,6 \%(-1,6$ a 4,$8 ; p=0,3)$ & 55,05 & 67,15 & $1,6 \%(0,6$ a 2,$7 ; p<0,01)$ & 45,72 & 50,89 & $0,3 \%(-0,7$ a 1,$3 ; p=0,2)$ \\
\hline $60+$ & 182,72 & 428,16 & $4,3 \%(3,8$ a 4,$9 ; p<0,01)$ & 177,36 & 308,36 & $2,9 \%(1,7$ a 4,$0 ; p<0,01)$ & 177,50 & 236,80 & $2,2 \%(1,7$ a 2,$7 ; p<0,01)$ \\
\hline
\end{tabular}




\section{Referências}

1. Guimarães RM, Andrade SSCA, Machado EL, Bahia CA, Oliveira MM Jacques FVL. Diferenças regionais na transição da mortalidade por doenças cardiovasculares no Brasil, 1980 a 2012. Rev Panam Salud Publica. 2015; 37(2):83-9.

2. Vasconcelos AMN, Gomes MMF. Transição demográfica: a experiência brasileira. Epidemiol Serv Saúde, Brasília. 2012; 21(4):539-48.

3. Santos JP, Paes NA. Associação entre condições de vida e vulnerabilidade com a mortalidade por doenças cardiovasculares de homens idosos do Nordeste. Rev Bras Epidemiol. 2014; 17(2):407-20.

4. World Health Organization (WHO). Health statistics and information systems. Estimates for 2000-2016. [Online]. Genebra. 2018. [Acesso em julho de 2019]. Disponível em: http://www.who.int/healthinfo/global burden_disease/estimates/en/index1.html.

5. Ministério da Saúde (BR). Sistema de informações sobre mortalidade (SIM) [Acesso em 13 de setembro de 2018]. Disponível em http://datasus.saude. gov.br/.

6. Lotufo PA, Fernandes TG, Bando, DH, Alencar AP, Benseñor, IM. Income and heart disease mortality trends in Sao Paulo, Brazil, 1996 to 2010. Int J Cardiol. 2013; 167(6):2820-3.

7. Gaui EM, Klein CH, Oliveira GMM. Mortalidade proporcional por insuficiência cardíaca e doenças isquêmicas do coração nas regiões do brasil de 2004 a 2011. Rio de Janeiro. Arq Bras Cardiol. 2016; 107(3):230-8.

8. Ribeiro ALP, Duncan, BB, Brant LCC, Lotufo PA, Mill JG, Barreto SM, et al. Cardiovascular health in Brazil trends and perspectives. American Heart Association, Circulation. 2016; 133:422-33.

9. Perricone AJ, Heide RSV. Novel therapeutic strategies for ischemic heart disease. Pharmacol Research 89 (2014) 36-45.

10. Alves LC, Leimann BCQ, Vasconcelos MEL, Carvalho MS, Vasconcelos AGG, et al. The effect of chronic diseases on functional status of the elderly living in the city of São Paulo, Brazil. Cad. Saúde Pública, Rio de Janeiro, 23(8):1924-30.

11. Simão $M$, Nogueira MS, Hayashida $M$, Cesarino EJ. Doenças cardiovasculares: perfil de trabalhadores do sexo masculino de uma destilaria do interior paulista. Rev Eletr Enfer. 2002; 4(2):27-35.

12. Magalhães FJ, Mendonça LBA, Rebouças CBA, Lima FET, Custódio IL, Oliveira SC. Fatores de risco para doenças cardiovasculares em profissionais de enfermagem: estratégias de promoção da saúde. Rev Bras Enferm. 2014; 67(3):394-400.

13. Sociedade Brasileira de Cardiologia. I Diretriz Brasileira de Prevenção Cardiovascular. Arq Bras Cardiol. 2013; 101(6):1-63.

14. Moraes IHS, Santos SRFR. Informações em saúde: os desafios continuam. Cienc Saúde Coletiva. 1998; 3(1):37-51

15. Instituto Brasileiro de Geografia e Estatística (IBGE). Sistema de Recuperação automática de dados-SIDRA. Acesso em 08 setembro 2018.Disponível em https://sidra.ibge.gov.br/home/pms/brasil.

16. Kim HJ, Fay MP, Feuer EJ, Midthune DN. Permutation tests for joinpoint regression with applications to cancer rates. Stat Med. 2000;19(3):335-51

17. Soeiro AM, Barros e Silva PGM, Roque EAC, Bossa AS, Biselli B, Leal TCAT, et al. Diferenças prognósticas entre homens e mulheres com síndrome coronariana aguda, dados de um registro brasileiro. Arq Bras Cardiol. 2018; 111(5):648-53.

18. Brant LCC, Nascimento BR, Passos VMA, Duncan BB, Benseñor IJM, Malta DC, et al. Variações e diferenciais da mortalidade por doença cardiovascular no Brasil e em seus estados, em 1990 e 2015: estimativas do Estudo Carga Global de Doença. Rev Bras Epidemiol. 2017; 20:116-28.

19. Mansur AP, Favarato D. Mortalidade por doenças cardiovasculares em mulheres e homens nas cinco regiões do Brasil, 1980-2012. Arq Bras Cardiol. 2016; 107(2):137-46.
20. Massa KHC, Duarte YAO, Filho ADPC. Análise da prevalência de doenças cardiovasculares e fatores associados em idosos, 2000-2010. Cienc Saúde Coletiva. 2019; 24(1):105-14

21. Sousa ALL, Batista SR, Sousa AC, Pacheco JAS, Vitorino PVO, Pagotto V. Prevalência, tratamento e controle da hipertensão arterial em idosos de uma capital brasileira. Arq Bras Cardiol. 2019; 112(3):271-8.

22. Enes CC, Slater B. Obesidade na adolescência e seus principais fatores determinantes. Rev Bras Epidemiol 2010; 13(1):163-71.

23. Kuschnir MCC, Bloch KV, Szklo M, Klein CH, Barufaldi LA, Abreu GA, et al ERICA: prevalência de síndrome metabólica em adolescentes brasileiros. Rev Saúde Pública. 2016; 50:11s.

24. Faria-Neto JR, Bento VFR, Baena CP, Olandoski M, Gonçalves LGO, Abre GA, et al. ERICA: prevalência de dislipidemia em adolescentes brasileiros. Rev Saúde Pública. 2016; 50:10s.

25. Ribas SA, Silva LCS. Dislipidemia em escolares na rede privada de Belém. Arq Bras Cardiol. 2009; 92(6):446-51.

26. Radaelli G, Sausen G, Cesa CC, Portal VL, Pellanda LC. Dislipidemia secundária em crianças obesas - há evidências para tratamento farmacológico? Arq Bras Cardiol. 2018; 111(3):356-61.

27. Machado APMC, Santos ACG, Carvalho KKA, Gondim MPL, Bastos NP, Rocha JVS, et al. Avaliação da adesão ao tratamento de pacientes com diabetes mellitus e seus fatores associados. REAS. 2019; 19:e565.

28. Mansur AP, Favarato D. Tendências da taxa de mortalidade por doenças cardiovasculares no Brasil, 1980-2012. Arq Bras Cardiol. 2016; 107(1):20-

29. Gaui EG, Oliveira GMM, Klein CH. Mortalidade por insuficiência cardíaca e doença isquêmica do coração no Brasil de 1996 a 2011. Arq Bras Cardiol. 2014; 102(6):557-65

30. Ishitani LH, Franco GC, Perpétuo IHO, França E. Desigualdade social e mortalidade precoce por doenças cardiovasculares no Brasil. Rev Saúde Pública 2006; 40(4):684-91.

31. Soares GP, Brum JD, Oliveira GMM, Klein CH, Souza e Silva NA et al. Evolução de indicadores socioeconômicos e da Mortalidade Cardiovascular em três Estados do Brasil. Arq Bras Cardiol. 2013; 100(2):147-56

32. Instituto de Pesquisa Econômica Aplicada (IPEA). Atlas de vulnerabilidade social. Disponível em http://ivs.ipea.gov.br/index.php/pt/. Acesso em: 10 agosto, 2019

33. Andrade L, Lynch C, Barbeito A, Spiecker EM, Vissoci JR, et al. Spatial distribution of ischemic heart disease mortality in Rio Grande do Sul, Brazil: ACM digital library, 2013. p.1-17.

34. Andrade Ld, Zanini V, Batilana AP, Carvalho ECAd, Pietrobon R, Nihe OK, Carvalho MDB. Regional disparities in mortality after ischemic heart disease in a brazilian state from 2006 to 2010. PLoS ONE.2013; 8(3): e59363

35. Ribeiro AG, Cotta RMM, Ribeiro SMR. A promoção da saúde e a prevenção integrada dos fatores de risco para doenças cardiovasculares. Cienc Saúde Coletiva. 2012; 17(1):7-1.

36. Lentsck MH, Saito AC, Mathias TAF. Tendência de declínio da hospitalizações por doenças cardiovasculares sensíveis à atenção primária. Texto Contexto Enferm, 2017; 26(2):e03170015.

37. Villela, LCM, Gomes FE, Meléndez JGV. Tendência da mortalidade po doenças cardiovasculares, isquêmicas do coração e cerebrovasculares. Rev enferm UFPE (on line) Recife2014;, 8(9): 3134-41

38. Álvares J, Guerra Junior AA, Araújo VE, Almeida AM, Dias CZ, Oliveira BA, et al. Acesso aos medicamentos pelos usuários da atenção primária no Sistema Único de Saúde. Rev Saúde Pública. 2017;51 (Supl 2):20s. 


\section{Artigo Original}

39. Nascimento RCRM, ÁlvaresJ, Guerra Junior AA, Gomes IC, Costa EA, Leite SN, et al. Disponibilidade de medicamentos essenciais na atenção primária do Sistema Único de Saúde. Rev Saúde Pública. 2017;51 (Supl 2):10s.

40. Santo AH. Causas mal definidas de morte e óbitos sem assistência. Rev Assoc Med Bras 2008; 54(1): 23-8.

41. Nogueira MC, Ribeiro LC, Cruz OG. Desigualdades sociais na mortalidade cardiovascular precoce em um município de médio porte no Brasil. Cad Saúde Pública. 2009; 25(11):2321-32.
42. Carvalho FCD, Vasconcelos TB, Arruda GMMS, Macena RHM. Modificações nos indicadores sociais da região Nordeste após a implementação da atenção primária. Trab Educ Saúde. 2019; 17(2):e0018925.

43. Soares GP, Brum JD, Oliveira GMM, Klein CH, Souza e Silva NA. Mortalidade por todas as causas e por doenças cardiovasculares em três estados do Brasil, 1980 a 2006. Rev Panam Salud Publica. 2010; 28(4):258-66. 